\title{
Comparison of manual versus robot-assisted contralateral gate cannulation in patients undergoing endovascular aneurysm repair
}

Original article with the following authors:

Sheena Cheung, MBBS$^{1}$ and Rafid Rahman, MBBS$^{1}$ (Joint first authors)

sheena.sl.cheung@outlook.com

rafid_rahman@hotmail.co.uk

Colin Bicknell, MD, FRCS ${ }^{1,2}$

Colin.bicknell@imperial.ac.uk

Danail Stoyanov, $\mathbf{P h D}^{3}$

danail.stoyanov@ucl.ac.uk

Ping-Lin Chang, $\mathbf{P h D}^{3}$

ping-lin.chang@ucl.ac.uk

Mimi Li, MBBS ${ }^{1}$

mimi.mzl.li@gmail.com

Alexander Rolls, MBBS, PhD $^{1}$

alex.rolls1981@googlemail.com

Liesbeth Desender, MD, PhD ${ }^{4}$

Liesbeth.desender@ugent.be

Isabelle Van Herzeele, $\mathrm{MD}, \mathrm{PhD}^{4}$

vhisabelle@gmail.com

Mohamad Hamady, MD FRCR ${ }^{2}$

m.hamady@imperial.ac.uk 
Celia Riga, MD, FRCS ${ }^{1,2}$ (corresponding author)

c.riga@imperial.ac.uk

1003 Queen Elizabeth the Queen Mother Wing (QEQM)

St Mary's Hospital

Praed St

Paddington

London W2 1NY

${ }^{1}$ Division of Surgery and Cancer, Imperial College London, United Kingdom

${ }^{2}$ Imperial Vascular Unit, Imperial College Healthcare NHS Trust, London, United Kingdom

${ }^{3}$ Centre for Medical Image Computing, University College London, United Kingdom

${ }^{4}$ Department of Thoracic and Vascular Surgery, Ghent University Hospital, Belgium 


\section{Abstract}

\section{Purpose}

Robotic endovascular technology may offer advantages over conventional manual catheter techniques. Our aim was to compare the endovascular catheter path-length (PL) for robotic versus manual contralateral gate cannulation during endovascular aneurysm repair (EVAR), using video motion analysis (VMA).

\section{Methods}

This was a multicentre retrospective cohort study with fluoroscopic video recordings of 24 EVAR cases (14 robotic, 10 manual) performed by experienced operators ( $>50$ procedures), obtained from four leading European centres. Groups were comparable with no statistically significant differences in aneurysm size $(\mathrm{p}=0.47)$ or vessel tortuosity $(\mathrm{p}=0.68)$.

Two trained assessors used VMA to calculate the catheter PL during contralateral gate cannulation for robotic versus manual approaches.

\section{Results}

There was a high degree of inter-observer reliability (Cronbach's $\alpha>0.99$ ) for VMA. Median robotic PL was $35.7 \mathrm{~cm}$ [interquartile range, IQR (30.8-51.0)] versus $74.1 \mathrm{~cm}$ [IQR (44.3170.4)] for manual cannulation, $\mathrm{p}=0.019$. Robotic cases had a median cannulation time of 5.33mins [IQR (4.58-6.49)] versus 1.24mins [IQR (1.13-1.35)] in manual cases $(\mathrm{p}=0.0083)$. Generated efficiency ratios (PL/aorto-iliac centrelines) was 1.6 (1.2-2.1) in robotic cases versus $2.6(1.7-7.0)$ in manual, $\mathrm{p}=0.031$.

\section{Conclusion}

Robot-assisted contralateral gate cannulation in EVAR leads to decreased navigation path lengths and increased economy of movement compared with manual catheter techniques. The benefit could be maximised by prioritizing robotic catheter shaping over habituated reliance on guide wire manipulation. Robotic technology has the potential to reduce the endovascular footprint during manipulations even for experienced operators with the added advantage of zero radiation exposure.

\section{Key words:}

endovascular, innovation, aneurysm, robotics. 


\section{Disclosures and declarations}

\section{Funding}

This research did not receive any specific grant from funding agencies in the public, commercial, or not-for-profit sectors

\section{Conflicts of interest}

All authors have read and approved this manuscript and there are no conflicts of interests.

\section{Ethical Approval}

This study was approved by the respective Ethics Committees in each of the institutions involved and in accordance with the ethical standards of the 1964 Helsinki Declaration and its later amendments.

\section{Consent}

Informed consent was obtained from all study participants.

\section{Availability of data}

Available on request

\section{Code Availability}

Not applicable 


\section{Introduction}

Endovascular robotic technology with remote steering provides an alternative to conventional manual catheter techniques. Flexible robotics may overcome limitations of manual devices such as: pre-determined and restricted catheter shapes and sizes; difficulties with catheter tip manoeuvrability, and instability for steering and treatment delivery. Pre-clinical studies have identified potential advantages in terms of accuracy and stability during endovascular manipulations (1-8). Clinical experience with robotic catheter technology is increasing, with published reports and early clinical series for aortic and peripheral vascular disease (9-16).

Remote catheter manipulation utilising robotic technology has also attracted interest from the intervernnational community due to the potential for occupational radiation reduction. However, comparative studies evaluating the clinical performance of flexible robotics against manual techniques remain scarce. Video motion analysis (VMA) during procedures describes the post-hoc evaluation of intra-operative movements. It has been utilised in surgical interventions to establish learning curves for surgical competence and provides objective evaluation of operator skill (17-19). For catheter-based interventions, VMA utilizes fluoroscopic sequences to derive motion-descriptive data on catheter and guide-wire movement (20).

Contralateral gate cannulation in endovascular aneurysm repair (EVAR) refers to the navigation of a catheter through the contralateral arterial tree and subsequently through the already-deployed opening ('gate') in the main body of the stent. This allows for the deployment of the contralateral limb- an essential step for successful aneurysm exclusion. Although rarely a challenge for the experienced interventionalist, it can impact on procedural flow in the presence of complex and tortuous patient anatomy (21). For the purpose of this study however, it serves as a reliable assessment of direct comparison between robotic and manual techniques. This is the first clinical study to objectively compare catheter path-length (PL) measurements for robotic versus manual techniques during contralateral gate cannulation in EVAR.

Our aim was to compare the endovascular catheter PL for robotic versus manual contralateral gate cannulation during EVAR, using video motion analysis. 


\section{Materials and methods}

\section{Magellan Robotic system}

The Magellan ${ }^{\mathrm{TM}}$ Robotic System (Hansen Medical, Mountain View, CA, USA) is designed for navigating the arterial tree. It consists of a workstation away from the $\mathrm{X}$-ray source and a robotic arm by the patient's bedside that delivers a co-axial robotic catheter system (catheter and sheath). It is an electromechanical master-slave system whereby the operator controls the device remotely from the workstation. In addition, an in-built wire manipulator facilitates remote control of conventional hydrophilic guide wires that pass through the robotic catheter lumen. A full description of the system is provided in previous publications (22).

\section{VMA Software}

Cathtrack, the VMA software used in the analysis was created using $\mathrm{C}++$ programming language and OpenCV (Open Source Computer Vision programming functions) library for frame-by-frame real time computer vision. It has been validated previously and can be used to accurately track catheter and guide wire movement post-hoc using fluoroscopic sequences (Figure 1) (23). The assessor uploads fluoroscopic video files onto the program and selects defined points to allow instrument tracking through consecutive frames. The software is semiautomatic- requiring a trained assessor for analysis and correction of errors due to image resolution reduction or angiographic movement. Cathtrack outputs 2D pixel coordinates (x,y) of the tracked point on a frame-by-frame basis to allow for data manipulation processing and analysis. Functions derived from Pythagoras' theorem can be used to calculate the total distance travelled by the tracked point i.e. the path-length (PL) measurement. It has been demonstrated in previous publications that VMA-derived PL is able to distinguish levels of experience in operators performing carotid artery stenting (23).

\section{Patient population \& Procedural details}

High quality, anonymised fluoroscopic images of 24 EVAR cases (14 robotic and 10 manual) were obtained from four leading European centres. All cases had anatomical landmarks clearly visible to allow VMA tracking, with no external interference obscuring fluoroscopic view, and full visibility of the guidewire $(\mathrm{GW})$ throughout contralateral gate cannulation. There were three operators used in manual cases and three (separate) robotic operators, all of whom had $>50$ procedures experience in their respective modality. 
For manual cases, EVAR was completed in the conventional manner. Due to technical limitations, current practice for EVAR using the Magellan system involves a hybrid procedure where initial access, main stent deployment and contralateral access is undertaken manually. The manual catheter is then exchanged with a robotic catheter to allow contralateral gate cannulation (which is achieved purely through robotic technique).

Informed consent and institutional review board permission was obtained for all patients as per standard guidelines for each institution. The contralateral gate cannulation stage was isolated for video motion analysis. Patient demographics can be seen in Table 1 .

\section{Tracking and Data Processing}

Tracking and data processing was performed by two trained assessors focusing on contralateral gate cannulation. The tracking point for producing the $2 \mathrm{D}$ coordinates was defined as the part of the instrument that was most distal, either the distal end of the guide wire or the distal end of the catheter tip. Tracking was standardised with pre-defined start to end points: starting with the point at which the wire-catheter tip crossed the pelvic brim in the anteroposterior (AP) view and finishing when the tip traversed the superior aspect of the proximal landing zone. Where a pigtail had already been inserted on the contralateral side before, the start-point used was the guidewire crossing the pelvic brim and normal protocol resumed once catheter manipulation began.

\section{Path length}

The PL was calculated in pixel units once the fluoroscopic sequence had been tracked. The pixel PL data were further processed to account for intra-operative magnification changes and $\mathrm{C}$-arm rotation, excluding movement artefact, to produce distance travelled (19). Anatomical measurements for individual patient's L4 vertebral height were obtained using pre-operative computed tomography (CT) and post-processing was performed using the OsiriX DICOM viewer (Pixmeo, Geneva, Switzerland). The measured bony landmarks were used to produce patient-specific, calibrated references in centimetres (Figure 2). The same bony landmarks were measured within fluoroscopic sequences in pixels at every intra-operative magnification segment using Cathtrack to allow for conversion of pixel PL into actual length in centimetres (Figure 2). This allowed calculation of a cumulative distance in centimetres representing the PL. 
Pre-operative CT images were analysed in OsiriX DICOM viewer to calculate the vessel central luminal length or centreline (CL) as an idealised optimal instrument path. Centrelines were produced using the distance from the ipsilateral iliac vessel traversing pelvic brim on anteroposterior view to the origin of the lower renal artery, through utilisation of threedimensional (3D) curved planar reformation (CPR) post-processing technique within the OsiriX software (Figure 2).

\section{Cannulation time}

Cannulation time was measured within the VMA software by multiplying the total number of frames by duration per unit frame- to give total duration from initiation of cannulation.

\section{Efficiency ratio}

To assess the efficiency of contralateral gate cannulation during EVAR, the ratio of path length distance to the idealized vessel centreline distance was calculated using the following equation: Efficiency Ratio $=($ Path Length $(\mathrm{cm})) /($ Centreline $(\mathrm{cm}))$. To evaluate robotic manipulation with regard to efficiency of use, additional VMA analysis was performed for robotic cases with the point of tracking at the distal tip of the guide wire $(\mathrm{GW})$ only.

\section{Case population characteristics}

Anatomical data on aneurysm size was available for all cases as part of pre-operative planning. Aorto-iliac tortuosity index was calculated using the method described by Chaikof and colleagues (24) using pre-operative CT scan data to establish a ratio of centerline distance to straight-line distance between start and end points. Tortuosity index relationship to anatomical severity grading is demonstrated in Table 2.

\section{Intra and Inter-observer reliability}

Six cases were re-analysed (with blinding to the original results) by each assessor to measure the intra-observer reliability of the tracking process.

Inter-observer reliability between the two assessors was determined through analysis of 5 cases of both robotic and manual groups. 


\section{Statistical Analysis}

Data analysis was carried out using Microsoft Excel 2013 and IBM SPSS Statistics version 22 (IBM Corp., Armonk, New York, USA). The data was assessed for normality using a ShapiroWilks test and found to be non-parametric. Mann-Whitney U and Spearman's rank correlation were used to determine significance and correlation strength respectively. A p-value of $<0.05$ was the threshold for statistical significance. Cronbach's a statistic was calculated for intraand inter-observer reliability testing. 


\section{Results}

\section{Intra and Inter-observer reliability}

The Cronbach Alpha scores for intra and inter-observer reliability were 0.999 and 0.991 respectively- indicating an excellent level of consistency.

\section{Case Population comparison}

There was no significant difference in patient characteristics between groups. Median aneurysm size for the robotic group was 59.0mm [interquartile range, IQR (55.5-68.0 5)] vs $57.0 \mathrm{~mm}$ [IQR (53.5-63.3)] in the manual group ( $\mathrm{p}=0.472)$. Median aorto-iliac tortuosity for the robotic group was 1.24 [IQR (1.17-1.30)] vs 1.26 [IQR (1.23-1.27)] in the manual group $(\mathrm{p}=0.682)$. There were only very weak $(\mathrm{Rho}=0.00-0.19)$ correlation between PL and tortuosity in both manual $(\mathrm{Rho}=-0.013)$ and robotic $(\mathrm{Rho}=0.171)$ case series.

\section{Robotic vs Manual analysis}

Median PL for robotic contralateral gate cannulation cases was significantly shorter when compared with manual catheter and wire manipulation: 35.7cm [IQR (30.8-51.0)] versus 74.1 cm [IQR (44.3-170.4)] for manual cannulation, $\mathrm{p}=0.019$ (Figure 3).

For robotic cases, the median efficiency ratio was found to be 1.6 [IQR (1.2-2.1)] compared to a manual median of 2.6 [IQR (1.7-7.0)], p=0.031. This can be seen in-Figure 4. The red line represents a $1: 1$ ratio of catheter navigation to the patient's centreline. Figure 46 shows the comparison between the path length and efficiency ratio between groups. There was no significant difference in centreline distances between the groups: robotic median $24.7 \mathrm{~cm}$ [IQR (22.2-28.2)] vs manual median $25.1 \mathrm{~cm}$ [IQR (23.0-26.0)], $\mathrm{p}=0.841$.

There was a statistically significant difference in cannulation times between groups, with the robotic group having a median cannulation time of 5.33mins [IQR (4.58-6.49) versus 1.24mins [IQR (1.13-1.35)] in the manual group ( $\mathrm{p}=0.0083)$.

\section{Subgroup analysis for Robotic Navigation}

To evaluate robotic manipulation with regards to efficiency of use, for each robotic caseadditional VMA analysis was performed with the point of tracking at the distal tip of the guide wire (GW) only. The median GW PL was $93.3 \mathrm{~cm}$ [IQR (61.3-170.2)] and the median GW 
efficiency ratio was 3.7 (2.7-20.7) (Figure 5). For every case, the GW PL and ER were significantly greater than the corresponding values obtained when tracking the robotic catheter $(p=0.001)$. Consequently, robotic cannulation was found to be technically less efficient when there is a greater reliance on GW manipulation and a relatively inactive robotic catheter tip. 


\section{Discussion}

The aim of this study was to compare robotic vs manual path length for contralateral gate cannulation in EVAR. When compared to manual cases, robotic cannulation demonstrated a statistically significant lower median PL, a narrower IQR and higher efficiency ratio with close conformity to the idealised PL.

The Magellan system is currently limited to only part of the EVAR procedure and contralateral gate cannulation was chosen as a standardised, comparable step of EVAR that is common to both robotic and manual techniques. It is a key step that can be technically challenging; especially in the presence of complex anatomy, with reported cases of failed cannulation attempts leading to increased fluoroscopy time and even the need for additional access $(19,25-$ 27).

This study utilised objective VMA analysis to provide a comparison between robotic and manual gate cannulation during EVAR. Whilst pre-clinical studies of endovascular robotic technology are numerous, there is a scarcity of data comparing clinical outcomes using validated metrics such as PL (1-6). The value of PL as a measure of skill has been demonstrated in a number of clinical studies, and it can measure competence and differentiate between operator experience levels in endovascular procedures $(19,23)$. Procedural time is another measure that has been shown to differentiate skill levels, experience and competency in minimally invasive surgery (28).

Despite the reduced PL, it is an interesting finding that median robotic cannulation time was significantly slower compared to manual (by 4.1 minutes, $\mathrm{p}=0.0083$ ). This may be explained by the increased PL found in all robotic cases when the point of tracking was the GW rather than the catheter itself ( $\mathrm{p}=0.001)$. GW shaping is an essential part of catheter manipulation in manual techniques as it allows catheter shaping/manoeuvring. However, the robotic catheter has a remote steering capability that should theoretically eliminate the need for any GW advances. One explanation for the discrepancy between robotic guidewire and catheter tip PL is that the operators were habituated to manual manoeuvres and employed these for robotic cannulation. 'Unlearning' these manoeuvres may form part of the learning curve in robotic manipulation and utilising robotic catheter shaping could increase efficiency, thus reducing cannulation time. 
The decreased PL and increased efficiency ratio in robotic cases may also be clinically significant in the context of reducing traumatic and embologenic manipulations in high-risk vascular beds $(8,22)$. Embologenic events, whilst rare in EVAR, may be catastrophic in other endovascular procedures and the role of robotic catheterisation in these is yet to be ascertained. The stable platform of the co-axial robotic system offers enhanced manoeuvrability and control; allowing the operator to steer, direct and 'drive' into the target while minimizing contact with the vessel wall $(4,29)$. The robotic catheter can also be used for initial angiography during EVAR to locate the lowest renal artery to streamline the procedure and reduce contrast load and manual pre-shaped catheter changes.

Another major advantage of robotic technology is the lack of radiation exposure for the operator (30). Recent evidence has shown that more complex EVAR procedures can lead to greater radiation exposure for all members in the operating room as well as a significant radiation dose during gate cannulation (31). The reduced variability, accurate navigation and catheter stability of robotic manipulation may favour more complex endovascular tasks (e.g. fenestrated EVAR and challenging embolisation targets) and thus reduce radiation exposure. The robotic workstation is now most-often located in the operating room behind a lead shield to help streamline the procedure, and the set up time of the apparatus has previously been measured as less than 2 minutes for experienced teams (15).

A realistic barrier to robotic access remains the initial cost of the system as well as the price of its consumables- limiting the uptake of this technology to a few specialist centres worldwide. Currently there are a number of robotic technologies available, with the Hansen platform representing the most advanced of these to date. However, the acquisition of Hansen Medical by Auris (Auris Health, Redwood City, CA, USA) means that the co-axial robotic system will no longer be commercially available until the next-generation platform launch.

\section{Study limitations}

A limitation of the VMA technique is that fluoroscopic analysis takes place in the twodimensional (2D) plane, and therefore does not account for any movement in the $\mathrm{Z}$ plane. However, validation studies have shown that $2 \mathrm{D}$ path length values tracked with Cathtrack show a linear relationship between 2D and 3D PL- suggesting that the amount of motion data lost in the $\mathrm{Z}$ plane is predictable and small $(4,19,32)$. The technique itself also requires significant assessor input and training due to its semi-automated nature. Automation may 
enable real-time tracking in live cases providing direct and simultaneous feedback to the operator. We hope to expand the use of VMA to investigate navigation techniques in other vascular beds.

Whilst there was no demonstrable difference between the anatomical and demographic factors in each group, there remains a possibility that non-measured variables could affect the differences in PL. For example, Figure 4 shows three manual cases having efficiency ratios greater than 5. Whilst these were from 3 different centres and operators, other variables may have had an impact. Studies with larger groups and measuring more variables will play an important role in providing a more comprehensive comparison between robotic and manual endovascular technology.

Using PL as a primary outcome measure for this stage of EVAR has shown promising resultsin a field where there is a paucity of clinical data comparing robotic vs manual modalities. However, there is a requirement for studies investigating additional clinical outcomes such as complication rate, radiation exposure and long-term outcomes. Reporting of current research and clinical experience from existing platforms will guide future iterations of the technology and we hope this will lead to studies comparing specific vascular beds in different procedures, $\doteqdot$ directing the clinical application of robotic endovascular technology.

\section{Conclusion}

Robot-assisted EVAR is feasible for contralateral gate cannulation with decreased navigation path lengths and increased economy of movement compared to manual catheter techniques. There is an associated increased cannulation time for robotic catheterisation compared to manual techniques, but the benefit could be maximised by prioritizing robotic catheter shaping over habituated reliance on guide wire manipulation. 


\section{References}

1. Riga CV, Bicknell CD, Rolls A, Cheshire NJ, Hamady MS. Robot-assisted Fenestrated Endovascular Aneurysm Repair (FEVAR) Using the Magellan System. J Vasc Interv Radiol. 2013;24(3):191-6.

2. Riga CV, Cheshire NJW, Hamady MS, Bicknell CD. The role of robotic endovascular catheters in fenestrated stent grafting. J Vasc Surg. 2010;51(4):810-20.

3. Riga C, Bicknell CD, Sidhu R, Cochennec F, Normahani P, Chadha P, Kashef E, Hamady M, Cheshire NJ. Advanced Catheter Technology: Is This the Answer to Overcoming the Long Learning Curve in Complex Endovascular Procedures. Eur J Vasc Endovasc Surg. 2011;42(4):531-8.

4. Riga CV, Bicknell CD, Hamady MS, Cheshire NJW. Evaluation of robotic endovascular catheters for arch vessel cannulation. J Vasc Surg. 2011;54(3):799-809.

5. de Ruiter QM, Moll FL, van Herwaarden JA. Current state in tracking and robotic navigation systems for application in endovascular aortic aneurysm repair. J Vasc Surg. 2015;61(1):256-64.

6. Bismuth J, Duran C, Stankovic M, Gersak B, Lumsden AB. A first-in-man study of the role of flexible robotics in overcoming navigation challenges in the iliofemoral arteries. $\mathrm{J}$ Vasc Surg. 2013;57(2 Suppl):14s-9s.

7. Cochennec F, Kobeiter H, Gohel M, Marzelle J, Desgranges P, Allaire E, Becquemin JP. Feasibility and safety of renal and visceral target vessel cannulation using robotically steerable catheters during complex endovascular aortic procedures. J Endovasc Ther. 2015;22(2):187-93.

8. Bismuth J, Kashef E, Cheshire N, Lumsden AB. Feasibility and safety of remote endovascular catheter navigation in a porcine model. J Endovasc Ther. 2011;18(2):243-9.

9. Carrell T, Dastur N, Salter R, Taylor P. Use of a remotely steerable "robotic" catheter in a branched endovascular aortic graft. J Vasc Surg. 2012;55(1):223-5.

10. Rolls AE, Riga CV, Bicknell CD, Regan L, Cheshire NJ, Hamady MS. Robot-Assisted Uterine Artery Embolization: A First-in-Woman Safety Evaluation of the Magellan System. J Vasc Interv Radiol. 2014;25(12):1841-8.

11. Bagla S, Smirniotopoulos J, Orlando JC, Piechowiak R. Robotic-Assisted Versus Manual Prostatic Arterial Embolization for Benign Prostatic Hyperplasia: A Comparative Analysis. Cardiovasc Intervent Radiol. 2017;40(3):360-5.

12. Chinnadurai P, Duran C, Al-Jabbari O, Abu Saleh WK, Lumsden A, Bismuth J. Value of C-Arm Cone Beam Computed Tomography Image Fusion in Maximizing the Versatility of Endovascular Robotics. Ann Vasc Surg. 2016;30:138-48.

13. Riga C, editor Safety, Feasibility and Value of the Magellan Endovascular Robot Based on Clinical Experience with 150 Cases. Veith Symposium; 2016 November 17, 2016. 
14. Lu T, Owji S, Chinnadurai P, Loh TM, Schwein A, Lumsden AB, Bismuth J. RoboticAssisted Coil Embolization of Ascending Aortic Pseudoaneurysm. Ann Thorac Surg. 2016;102(5):e451-e3.

15. Bonatti J, Vetrovec G, Riga C, Wazni O, Stadler P. Robotic technology in cardiovascular medicine. Nat Rev Cardiol. 2014;11(5):266-75.

16. Schwein A, Kramer B, Chinnadurai P, Virmani N, Walker S, O'Malley M, Lumsden $\mathrm{AB}$, Bismuth J. Electromagnetic tracking of flexible robotic catheters enables "assisted navigation" and brings automation to endovascular navigation in an in vitro study. J Vasc Surg. 2018;67(4):1274-81.

17. Egi H, Okajima M, Yoshimitsu M, Ikeda S, Miyata Y, Masugami H, Kawahara T, Kaneko M, Asahara T. Objective assessment of endoscopic surgical skills by analyzing direction-dependent dexterity using the Hiroshima University Endoscopic Surgical Assessment Device (HUESAD). Surg Today. 2008;38(8):705-10.

18. Duran C, Estrada S, O'Malley M, Lumsden AB, Bismuth J. Kinematics effectively delineate accomplished users of endovascular robotics with a physical training model. J Vasc Surg. 2015;61(2):535-41.

19. Rolls AE, Riga CV, Rahim S, Stoyanov DV, Van Herzeele I, Mikhail G, Hamady M, Cheshire NJ, Bicknell CD. Video motion analysis in live coronary angiography differentiates levels of experience and provides a novel method of skill assessment. EuroIntervention. 2017;13(12):e1460-e7.

20. Rolls AE, Riga CV, Bicknell CD, Stoyanov DV, Shah CV, Van Herzeele I, Hamady M, Cheshire NJ. A pilot study of video-motion analysis in endovascular surgery: development of real-time discriminatory skill metrics. Eur J Vasc Endovasc Surg. 2013;45(5):509-15.

21. Wolf YG, Tillich M, Lee WA, Rubin GD, Fogarty TJ, Zarins CK. Impact of aortoiliac tortuosity on endovascular repair of abdominal aortic aneurysms: evaluation of 3D computerbased assessment. J Vasc Surg. 2001;34(4):594-9.

22. Riga CV, Bicknell CD, Hamady M, Cheshire N. Tortuous Iliac Systems-A Significant Burden to Conventional Cannulation in the Visceral Segment: Is There a Role for Robotic Catheter Technology? J Vasc Interv Radiol. 2012;23(10):1369-75.

23. Rolls AE, Riga CV, Bicknell CD, Stoyanov DV, Shah CV, Van Herzeele I, Hamady M, Cheshire NJ. A Pilot Study of Video-motion Analysis in Endovascular Surgery: Development of Real-time Discriminatory Skill Metrics. Eur J Vasc Endovasc Surg. 2013;45(5):509-15.

24. Chaikof EL, Fillinger MF, Matsumura JS, Rutherford RB, White GH, Blankensteijn JD, Bernhard VM, Harris PL, Kent KC, May J, Veith FJ, Zarins CK . Identifying and grading factors that modify the outcome of endovascular aortic aneurysm repair. J Vasc Surg. 2002;35(5):1061-6.

25. Wilson WRW, Benveniste GL. EVAR Technical Tip - Confirmation of Contralateral Limb Gate Cannulation Using a Moulding Balloon. EJVES Extra. 2010;20(3):e25-e6. 
26. Georgakarakos E, Trellopoulos G, Ioannou CV, Tsetis D. Technical challenges encountered during deployment of the ovation abdominal aortic stent-graft system. J Endovasc Ther. 2014;21(2):333-8.

27. Maleux G, Houthoofd S, Fourneau I. Use of a Microcatheter to Cannulate the contralateral Gate in Endovascular Aortic Repair. Vasc Endovascular Surg. 2017. Doi:10.1177/1538574417697209.

28. Aggarwal R, Crochet P, Dias A, Misra A, Ziprin P, Darzi A. Development of a virtual reality training curriculum for laparoscopic cholecystectomy. Br J Surg. 2009;96(9):1086-93.

29. Rafii-Tari H, Riga CV, Payne CJ, Hamady MS, Cheshire NJ, Bicknell CD, Yang GZ. Reducing contact forces in the arch and supra-aortic vessels using the Magellan robot. J Vasc Surg. 2016;64(5):1422-32.

30. Li M, Riga C, Cheung S, Burfitt N, Thomas R, Jenkins M, Cheshire NJ, Hamady M, Bicknell CD. Occupational Radiation Exposure during FEVAR: A Stage-By-Stage Analysis. Br J Surg. 2015;102:23-23.

31. de Ruiter QM, Jansen MM, Moll F, Hazenberg C, Kahya N, van Herwaarden J. Procedure and step-based analysis of the occupational radiation dose during endovascular aneurysm repair in the hybrid operating room. J Vasc Surg. 2018 Jun; 67(6): 1881-1890.

32. Mung JC, Huang SG, Moos JM, Yen JT, Weaver FA. Stereotactic endovascular aortic navigation with a novel ultrasonic-based three-dimensional localization system. J Vasc Surg. 2013;57(6):1637-44. 


\section{Tables}

Table 1: Patient Demographics

\begin{tabular}{|c|c|c|}
\hline & Robotic $(n=14)$ & Manual $(n=10)$ \\
\hline Median Age in Years (IQR) & $72.5(64.8-79.5)$ & $74.5(68.0-80.8)$ \\
\hline Male Gender & $13(93 \%)$ & $10(100 \%)$ \\
\hline Smoker & $12(86 \%)$ & $2(20 \%)$ \\
\hline Hypertension & $13(93 \%)$ & $9(90 \%)$ \\
\hline Diabetic & $4(29 \%)$ & $2(20 \%)$ \\
\hline Renal Impairment & $6(43 \%)$ & $1(10 \%)$ \\
\hline $\begin{array}{l}\text { Median Aneurysm size in } \mathrm{mm} \\
(\mathrm{IQR})(\mathrm{p}=0.472)\end{array}$ & $59.0(55.5-68.5)$ & $57.0(53.5-63.3)$ \\
\hline
\end{tabular}

Table 2. Severity grading for tortuosity index with number of corresponding cases in each group.

\begin{tabular}{|l|l|l|l|}
\hline Severity Grading & Tortuosity Index (T) & Robotic (n) & Manual (n) \\
\hline Absent & $\mathrm{T} \leq 1.15$ & 3 & 1 \\
\hline Mild & $1.15<\mathrm{T} \leq 1.325$ & 8 & 7 \\
\hline Moderate & $1.325<\mathrm{T} \leq 1.4$ & 0 & 0 \\
\hline Severe & $\mathrm{T}>1.4$ & 3 & 2 \\
\hline Median tortuosity index $(\mathrm{p}=0.682)$ & 1.24 & 1.26 \\
\hline
\end{tabular}




\section{Legends for illustrations}

Fig. 1 Cathtrack video motion tracking software interface

Fig. 2 Anatomical measurements of vertebral height and centreline distance done on patient specific pre-operative CT scans with processing done in OsiriX DICOM viewer; top insert shows vertebral distance measured in VMA software by pixels to generate ratio allowing for translation of pixel to cm units.

Fig. 3 Box-and-whiskers plots of manual versus robotic path length values, with representative case examples for each group shown to the right (manual tortuosity 1.21, robotic tortuosity 1.22)

Fig. 4 Schematic of the robotic efficiency ratios manual vs robotic data distributed around 1:1 ratio (red line) of distance equal to centreline distance.

Fig. 5 Boxplot comparison of efficiency ratios between robotic catheter (RC) tracking and $\mathrm{GW}+\mathrm{RC}$ tracking showing significant difference between the two groups. $(\mathrm{p}=0.001)$.

Fig. 6 Scatter graph comparing path length to efficiency ratio for both manual and robotic cases. 